\title{
Automated Detection and Classification of Fundus Diabetic Retinopathy Images using Synergic Deep Learning Model
}

\author{
Shankar K ${ }^{\mathrm{a},}$, Abdul Rahaman Wahab Sait ${ }^{\mathrm{b}}$, Deepak Gupta ${ }^{\mathrm{c}}$, Lakshmanaprabu S.K ${ }^{\mathrm{d}}$, Ashish Khanna ${ }^{\mathrm{c}}$, and \\ Hari Mohan Pandey \\ ${ }^{a}$ Department of Computer Applications, Alagappa University, Karaikudi, India; shankarcrypto@ gmail.com

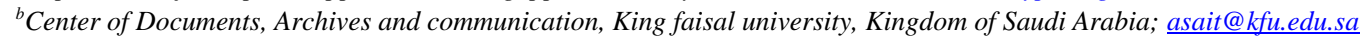 \\ ${ }^{c}$ Maharaja Agrasen Institute of Technology, Delhi, India.Email: deepakgupta@mait.ac.in, ashishkhanna@mait.ac.in \\ ${ }^{d}$ Department of Electronics and Instrumentation Engineering, B. S. Abdur Rahman Crescent Institute of Science and Technology, Chennai, India. Email: \\ prabusk.leo@gmail.com \\ eEdge Hill University, UK. Email: pandeyh@edgehill.ac.uk
}

\begin{abstract}
At present days, DR becomes a more common disease affecting the eyes because of drastic rise in the glucose level of blood. Almost half of the people under the age of 70's get severely affected due to diabetes. The earlier recognition and proper medication results to loss of vision in several DR patients. When the warning signs are identified, the severity level of the disease has to be validated to take decisions regarding the proper treatment. The current research focuses on the concept of classifying the images of DR fundus based on the severity level using a deep learning model. This paper proposes a deep learning based automated detection and classification model for fundus diabetic retinopathy (DR) images. The proposed method involves several processes namely preprocessing, segmentation and classification. Initially, preprocessing stage is carried out to get rid of the unnecessary noise exist in the edges. Next, histogram based segmentation takes place to extract the useful regions from the image. Then, synergic deep learning (SDL) model is applied to classify DR fundus images to various severity levels. The justification of the presented SDL model is carried out on Messidor DR dataset. The experimentation indicated that the presented SDL model offers better classification over the existing models.
\end{abstract}

Keywords: Classification, Deep learning, Diabetic Retinopathy, Messidor dataset. 


\section{Introduction}

Diabetes becomes a more common disease among people all over the globe due to the rise in the level of glucose in the blood. When the irregularity in glucose level continues for a longer duration, the blood vessels will be severely damaged. The person who suffers from diabetes has a great chance of having kidney problems, eye injury, gum bleeding and nerve injuries. At the same time, there is a high risk of heart problems in diabetic patients. Based on the organs which are damaged by the increased glucose level, the disease will be named. In this study, we focus on the disease of diabetic retinopathy (DR) which indicates the injury at the retina of the eye. World Health Organization (WHO) predicted that the seventh lethal disease is diabetes [1]. A statistical report revealed that around 61.3 million under the age of $20-79$ is recognized as diabetic patients. It is also predicted that the count will be increased to approximately 102 million by the year 2030 [2]. A constant rise in blood glucose level surely affects the retina. This rise in glucose level severely damages the blood vessels, leads to blood leak in the eyes and also reduces the vision. The interesting behavior of the human body is it implicitly has the capability to cure itself. Once the blood leakage is identified, it will activate the neighboring cells to manage the scenario. This action results in an irregular increase in extra blood vessels and recently grown blood vessels are not strong [3]. It affects the people's vision in a short time. Therefore, it is very important that a diabetic patient has to take an eye tests at a uniform time. In generally, Fundus photography is an important eye testing method employed for the diagnosis of diseases at the earlier level.

The severity of diseases is generally identified by the existence of abnormality and its size. The detection of manifestations like venous beading, microaneurysms, hemorrhage is an important procedure in the testing process. The Micro aneurysms indicate the size of the blood clot of around $100-120 \mu \mathrm{m}$ which is generally in the shape of a circle. The blood leakage from a damaged blood vessel is known as hemorrhage. The irregular development of miniature blood vessels is called neovascularization. Next, the venous beading indicates the fundamental expansion of the veins placed next to the occluded arterioles. DR can be classified into Non-Proliferative Diabetic Retinopathy (NPDR) and Proliferative Diabetic Retinopathy (PDR). Also, based on the severity level of the disease, the NPDR is again classified into different stages.Sample DR images indicating various stages are illustrated in Fig. 1.
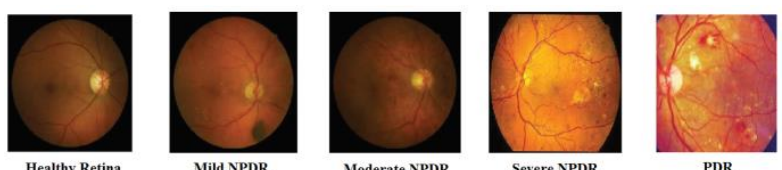

Fig. 1. Stages involved in DR.

DR is an important ailment and could be saved from losing the vision when the disease is identified at the beginning level. When the disease is identified, then the patient has to be validated for every six months to recognize the disease progresses [4]. A novel technique is developed to detect as well as classify the fundus images which finds useful to eradicate the loss of vision because of DR. Several studies have presented various techniques for facilitating the accurate identification of DR. The identification and categorization of DR takes place through the segmentation of the parts of the fundus image or the examination of the fundus image for the incidence of exudates, lesions, micro aneurysms, and so on.

Singh and Tripathi [5] developed a model that determines diverse characteristics like abnormalities in the foveal zone and micro aneurysms. The presented model makes use of curvelet coefficients from the fundus images as well as angiogram. A 3stage classifier model is employed and the experimentation is done on a group of 70 patients. The presented model attained a maximum sensitivity value of $100 \%$. Authors [4] presented a DR image classification model based on the existence of micro aneurysms. The characteristics like circularity and area of micro aneurysms are taken for extracting features. The dataset such asDIARETDB1, DRIVE and ROC are utilized in the study. The projected model attained maximum classifier results with the sensitivity and specificity of $94.44 \%$ and $87.5 \%$ correspondingly. The presented model makes use of principal component analysis (PCA) for segregating the image of the optic disc from fundus images. By the use of an improved MDD classification model, a classification result with an increase in $25-40 \%$ detection is achieved. A total of 39 images are utilized to carry out this work and a classification takes place indicating a total of only 4 normal and 35 abnormal images.

The author in [6] evaluated the experimental outcome of the trained support vector machine (SVM) on 3 benchmark dataset and achieved a higher accuracy of 93\%. Authors [7] explained the usage of texture features from a local binary patterns for detecting the exudates and reported that classification performance of $96.73 \%$ is found. Another dual classifier model is developed in [8]. It includes a bootstrapped decision tree for classifying the fundus images. A 2-binary vessel map is a generation by the reduction in the dimensionality of feature vectors.

A Gabor filtering and SVM classification model is applied in [9] for the categorization of DR images. Earlier to the usage of the classifier, Circular Hough Transform (CHT) and CLAHE models are provided to input images and attained a detection rate of $91.4 \%$ on the STARE dataset. The morphological operations utilizing the intensities of the images as threshold values to segment images are provided in [10]. Bhatkar and Kharat [11] explained the applications of $\mathrm{CNN}$ models with the data augmentation models in the classifier process of DR images. The severity level of DR undergoes 5 stages and is tested on the Kaggle dataset.

Partovi et al. [12] introduced a model of using an error based autonomous networks to classify the images. It is validated on the dataset containing the remote sensing images. Deep CNN (DCNN) model offered an extensive feature extractionclassification technique for classifying the medical images. Next, $\mathrm{Xu}$ et al. [13] make use of a DCNN method for minimizing the human annotations and develops better feature representation in a histopathological colon for classifying cancer image. Shen et al. [14] presented a method of multi-crop pooling and is employed to DCNN for capturing object salient details to classify the lung nodule on the CT images.

In [15], a multimodality neuroimaging and genetic data are applied for the identification of Alzheimer's disease (AD) and its prodromal status, Mild Cognitive Impairment (MCI), from normal aging subjects. Here, an effective three-stage deep feature learning and fusion model has been presented. In [16], deep learning based Dual-Supervised Fully Convolutional Networks called BIRNet is applied for image registration using the prediction of deformation from image appearance. In [17], important extensions and generalization to multiblock multiway (tensor) data are given. [18] presented a new technique called "strength and similarity guided sparse group representation (SSGSR)," which exploits both BOLD signal temporal correlation-based "low-order" functional connectivity (LOFC) and inter-subject LOFC-profile similarity-based "high-order" functional connectivity (HOFC). Some other deep learning models have also been presented in [19-23].

In this study, we focus on the concept of classifying the images of DR fundus based on the severity level. The key contributions of the current research are outlined as follows:

- We proposed a deep learning model for automated detection and classification of fundus diabetic retinopathy (DR) images. 
- We presented the working of the proposed model comprehensively. Initially, preprocessing stage is carried out for removing the useless noise occurs at the edges. Then, synergic deep learning (SDL) model is applied for the classification of DR fundus images into various stages of severity.

- Extensive experiments are conducted to evaluate the performance of the proposed deep learning model. The justification of the presented SDL model is carried out on the Messidor DR dataset.

- We showed comparative analysis of the proposed model against the state-of-the-art models. The experimental results indicated that the presented SDL model offers better classification over the existing models.

\section{The Proposed Model}

This study intends to the classification of DR fundus images with maximum detection rate. Since more number of patients is suffered by DR, it is essential to classify the patients into various DR stages in an effective way. The overall process is depicted in Fig. 2 and the processes are explained below.

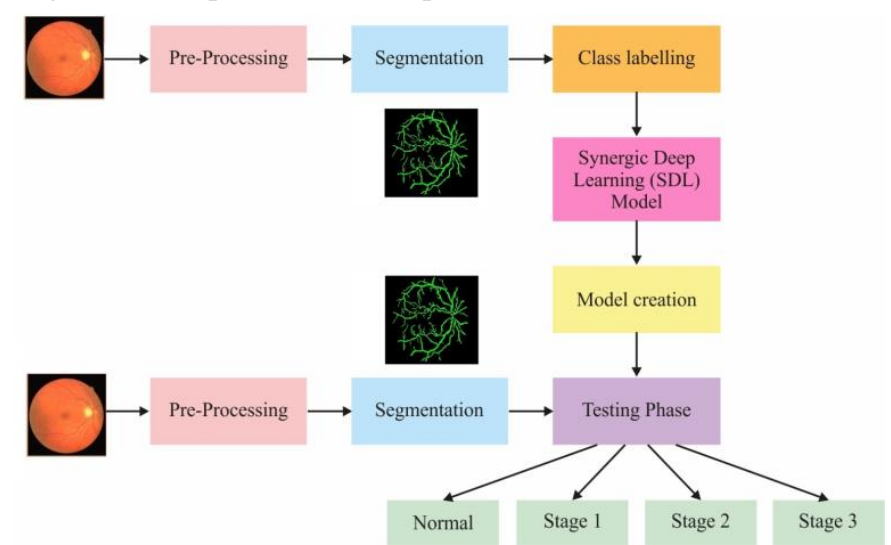

Fig. 2. The Proposed Model.

\subsection{Preprocessing}

A set of four stages namely healthy and stages 1-3 are present in the Messidor dataset. For every image, an annotation is provided in the Excel file which is employed to group the stages. Then, the green channel offers information related to the optical nerves and relevant characteristics of the retina. In the beginning, the images undergo partitioning to three RGB channels. In the next stage, histogram based segmentation takes place to extract the details in green color for further processing.

\subsection{Histogram based segmentation}

In this study, histogram based segmentation takes place on the DR images, which involve two levels. In the first level, the main colors present in the image are recognized and constructed the segmented image regions where the pixel in each portion is color to one between the colors over other colors. A Histogram $\mathrm{H}$ is generated in the first phase and complete peaks are identified. On comparing with the variable $\theta$, small $H$ peaks are not investigated. So, these peaks are unrecognized as dominant ones. In the second level, merging takes place based on the sizes of the segmentation region for minimizing the number of regions in the segmented image.

Despite the fact that when it is included through compelled occurrences, ignoring the number of peaks may deliver starting point in an image any place, not whole I pixels are designated towards the segmented regions. I pixels that could not be assigned to any region of segmentation are dealt with while in the next stage of the process. By thinking about the existence of whole peaks, $\theta$ value can be picked. The $\theta$ worth must be a little percent of peak occurrence peak value. The peak occurrences of arithmetic mean of $\mathrm{H}$ is demonstrated by $\mu$, the standard value is generated since variable $\theta$ in usually fulfilling results tentatively. To combine any voxels peak of $\mathrm{H}$, a second variable $\tau$ is utilized where colors have not fluctuated drastically from edge color. It takes place by the use of comparable representative color relationship by portraying any peak to whole $\mathrm{H}$ voxels which are joined with the edges. The $\tau$ value depends upon the maximal disparity where client acknowledges for colors to be consolidated. In 3D histogram, through the consideration of the maximal Euclidean separation between different colors and decreased colors are inferred to composite color image portrayal, and the standard value of $\tau=50$ is recommended. Hence, the peak pertinence is exceptionally constrained through the count that colors connected with the edge exist in the image I. The whole peaks that are identified are viewed as predominant. In any case, peaks in which the colors at related agent do not fluctuate unequivocally from each other may deliver to be joined into diverse groups. At the point when contrasted with recognized peak count, the end groups and representative colors are likely to be low.

At the point when $\tau$ is high, more chance is present to join with the peaks. The whole $\mathrm{H}$ voxels which include values differing from zero and good ways from peal centroid when contrasted with $\tau$ is joined to present peak. $H$ voxels that are connected to an edge are not considered while different edges are inspected. Simultaneously, enormous $\tau$ qualities may give converging of colors where the end user will distinguish as disparate one. The lower $\tau$ values not just make coming about images through over fragmented any place an enormous quantity of colors is utilized, nonetheless, it likewise has a hazard to leave gigantic information image I partition that isn't allocated to any zone. For I pixels, this may be a case at high separation when contrasted with $\tau$ from any edge found at $H$. To infer a predominant result, $\theta$ and $\tau$ determination is a noteworthy point. For each image, we found the unrivaled $\theta$ and $\tau$ values and infer the different rates by looking at the individual outcomes that converge in normal with prescribed default esteems. An image I is inspected when whole pinnacles had been found. Each I pixel of color $(\mathrm{x}, \mathrm{y}, \mathrm{z})$ is fixed as relating color connected with position $(\mathrm{x}, \mathrm{y}, \mathrm{z})$ voxel of $\mathrm{H}$. Along these lines, it is profoundly noteworthy to pick $\theta$ and $\tau$ values so as to that only a few pixels assembled or meager perhaps in small size regions which stays unallocated.

The resulting period of segmentation system centers at combining regions. To decrease over segmentation and to think about unassigned pixels, blending is performed. For various whole I partition areas, naming of associated segment is utilized, to begin with. The region is recorded for each associated part. Pixels that have a place with related component including little zone when contrasted with priori fixed worth $\gamma$ is appointed as zero out of one review of $\mathrm{I}$. The pixels are dispensed progressively to neighboring territory in that they have colossal neighbors. We suggest $\gamma$ default worth relying upon reproduction of segmentation areas. Consolidating may give a decrease in delegate color tallies. While I pixels offered delegate color, they are converged into related components highlighted through territory which is little when contrasted with $\gamma$. The number of segmentation area is enormous when contrasted with delegate color tallies.

\subsection{SDL based classification}

The projected SDL model is defined by SDL ${ }^{\mathrm{k}}$ includes a set of 3 major components namely input layer, k DCNN components and $\mathrm{C}_{\mathrm{k}}^{2}$ synergic network $(\mathrm{SN})$. The overall SDL model is depicted in Fig. 3. Each DCNN element of the network structure gives independent learning depiction from data with the direction of proper labels of input data. The SN includes a model of fully connected structure for ensuring whether the input layer comes under the similar classes and gives the remedial comment on the existence of a synergic error. Next, the SDL model undergoes partition into a set of 3 sub-modules. 


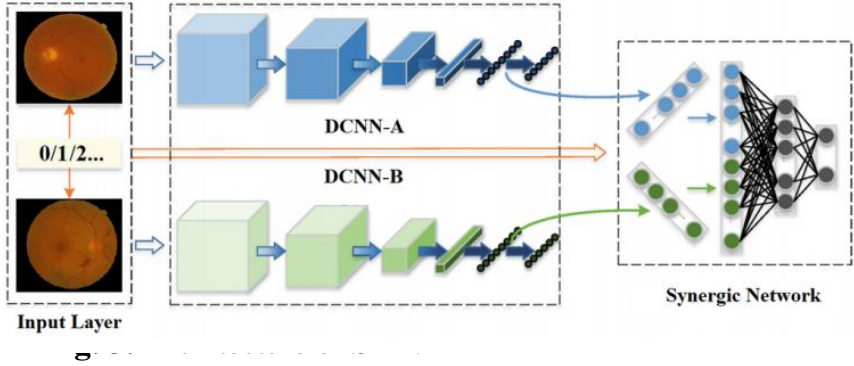

1. xPair Input Layer

Contrasting to classical DCNN, the projected $\mathrm{SDL}^{\mathrm{k}}$ model receives $\mathrm{n}$ input data concurrently that undergoes arbitrary choice from the training set. Each set of 200 data with the class label is provided as input to a DCNN components and every pair contains an equivalent synergic label given by the SN. To maintain uniform size, the images are resized into $224 \times 224 \times 3$ by the use of bicubic interpolation.

\section{DCNN Component}

Because of the nature of implicit ability of the significant residual network (ResNet), a ResNet -50 can be used to initialize each DCNN components as DCNN-a (a = $1,2, \ldots, n)$. However, it is noted that any DCNN, like AlexNet, GoogLeNet and VGGNet is placed in the SDL model as DCNN component. These components undergo training utilizing a data sequence $\mathrm{X}=\left\{\mathrm{X}^{(1)}, \mathrm{X}^{(2)}, \ldots, \mathrm{X}^{(\mathrm{M})}\right\}$ and a resulting class label series $Y=\left\{y^{(1)}, y^{(2)}, \ldots, y^{(M)}\right\}$, aiming to compute the group of variables $\theta$ that undergoes the reduction of the cross-entropy loss as represented here.

$$
\log (\theta)=-\frac{1}{M}\left[\sum_{a=1}^{M} \sum_{b=1}^{K} 1\left\{y^{(1)}=b\right\} \log \frac{e^{z_{b}^{(a)}}}{\sum_{l=1}^{K} z_{l}^{(a)}}\right]
$$

where $n$ indicates the class count, $Z^{(a)}=F\left(x^{(a)}, \theta\right)$ represents the forward computing. The obtained variable set for DCNN a is represented as $\theta^{\mathrm{a}}$, and the variables do not share between numerous CNN components.

\section{SDL model}

For further supervision of every DCNN component with the synergic labels of every data pair, an SN is implemented for fully connected learning, embedding and input layers. Consider $\left(\mathrm{Z}_{\mathrm{A}}, \mathrm{Z}_{\mathrm{B}}\right)$ as a data pair given as input to two DCNN components namely (DCNNa, DCNNb), correspondingly. The outline of SN is shown in Fig. 4. The outcome from the subsequent final fully connected layer in a DCNN indicates the deep data features undergoes learning by DCNN that can be derived from forward computing, as given below.

$$
\begin{aligned}
& \mathrm{f}_{\mathrm{A}}=\mathcal{F}\left(\mathrm{Z}_{\mathrm{A}}, \theta^{(\mathrm{a})}\right)(2) \\
& \mathrm{f}_{\mathrm{B}}=\mathcal{F}\left(\mathrm{Z}_{\mathrm{B}}, \theta^{(\mathrm{a})}\right)(3)
\end{aligned}
$$

Subsequently, the deep features from each data are incorporated by the use of $\mathrm{f}_{\mathrm{A}^{\circ} \mathrm{B}}$, and the particular result is the synergic label for data pair is represented as follows.

$$
y s\left(Z_{A}, Z_{B}\right)=\left\{\begin{array}{lll}
1 & \text { if } & y_{A}=y_{B} \\
0 & \text { if } & y_{A} \neq y_{B}
\end{array}\right.
$$

To avoid the challenge of unbalanced data, the percentage data pair exists in a class of each batch is around 45 to $55 \%$. It is simple to view the synergic signal through the enclosure of other sigmoid layer and succeeding binary cross entropy loss is represented below

$$
\mathrm{l}^{\mathrm{S}}\left(\theta^{\mathrm{S}}\right)=\mathrm{y}_{\mathrm{S}} \log \widehat{\mathrm{y}_{\mathrm{S}}}+\left(1-\mathrm{y}_{\mathrm{S}}\right) \log \left(1-\widehat{\mathrm{y}_{\mathrm{S}}}\right)
$$

where $\theta^{S}$ is the variable of the $\mathrm{SN}, \widehat{\mathrm{y}_{\mathrm{S}}}$ is the forward computing of the SN. It verifies that when the input data pair belongs to a similar category and provided the remedial response on the occurrence of the synergic error.

\section{Training and Testing}

On the completion of the training procedure, the parameter of DCNN components and SN can be updated as follows.

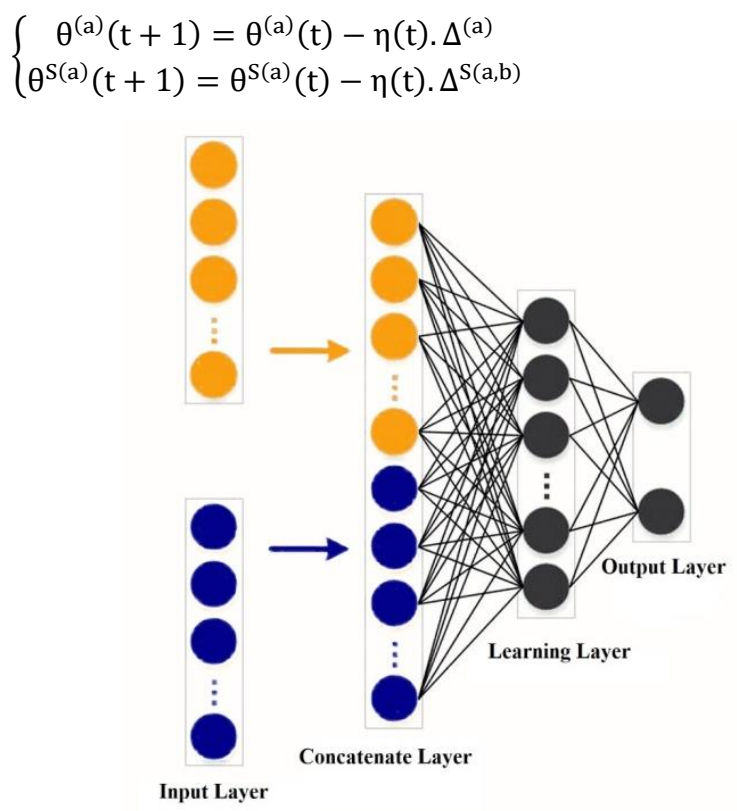

Fig. 4. Outline of SN.

where $\eta(t)$ and $S(a, b)$ represents learning rate and $S N$ between DCNNa and DCNNb is represented below.

$$
\begin{array}{r}
\Delta^{(\mathrm{a})}=\frac{\partial \mathrm{l}^{(\mathrm{a})}\left(\theta^{(\mathrm{a})}\right)}{\partial \theta^{(\mathrm{a})}}+\lambda \sum_{\mathrm{b}=1, \mathrm{~b} \neq \mathrm{a}}^{\mathrm{n}} \frac{\partial \mathrm{l}^{\mathrm{S}(\mathrm{a})}\left(\theta^{\mathrm{S}(\mathrm{a}, \mathrm{b})}\right)}{\partial \theta^{\mathrm{S}(\mathrm{a}, \mathrm{b})}} \\
\Delta^{\mathrm{S}(\mathrm{a})}=\frac{\partial \mathrm{l}^{\mathrm{S}(\mathrm{a})}\left(\theta^{\mathrm{S}(\mathrm{a}, \mathrm{b})}\right)}{\partial \theta^{\mathrm{S}(\mathrm{a}, \mathrm{b})}}
\end{array}
$$

and $\lambda$ indicates the trade-off between sub-version of classification as well as synergic error. The summary of the training procedure of the $\mathrm{SDL}^{2}$ model is enhanced. With the usage of trained SDL ${ }^{\mathrm{k}}$ model to classify the test data $\mathrm{x}$, each DCNN component DCNN - i offered a prediction vector $\mathrm{P}^{(\mathrm{a})}=$ $\left(\mathrm{p}_{1}^{(\mathrm{a})}, \mathrm{p}_{2}^{(\mathrm{a})}, \ldots, \mathrm{p}_{\mathrm{k}}^{(\mathrm{a})}\right)$, activation in the final fully connected layer. The class labels of the test data will undergo prediction as represented here.

$$
y(Z)=\underset{j}{\operatorname{argmax}}\left\{\sum_{i=1}^{k} p_{1}^{(i)}, \ldots, \sum_{i=1}^{k} p_{j}^{(i)}, \ldots, \sum_{i=1}^{k} p_{k}^{(i)}\right.
$$

\section{Performance Validation}

\subsection{Dataset description}

For the validation of the presented SDL model for the identification of DR, a benchmark MESSIDOR dataset is used [24]. This data has approximately 1200 color fundus images with proper annotation. The images in the dataset come under four categories. Image grading takes place on the existence of micro aneurysms and hemorrhages is allocated to the images. The image which exhibits no symptoms indicates the healthy retina. The image which has some microaneurysms represents stage 1 . The image which has some microaneurysms as well as hemorrhages comes under stage 2 and the images indicating more microaneurysms as well as hemorrhages comes under stage 3 . The information related to the dataset is shown in Table 1. For experimentation purposes, 10 fold cross-validation process is applied. 
Table 1. Dataset details

\begin{tabular}{|l|l|l|}
\hline DR stages & Details & Labels \\
\hline \hline Normal & No abnormality & Healthy \\
\hline Mild NPDR & Micro aneurysms & Stage 1 \\
\hline Moderate NPDR & Few Micro aneurysms & Stage 2 \\
Severe NPDR & $\begin{array}{l}\text { Venous Beading + intraretinal } \\
\text { Microvascular abnormality } \\
\text { (IRMA) }\end{array}$ & $\begin{array}{l}\text { Sitreous/ Pre-retinal } \\
\text { Hemorrhage }\end{array}$ \\
\hline PDR & & \\
\hline
\end{tabular}

\subsection{Performance Measures}

A set of evaluation parameters used to investigate the performance of the presented SDL model are sensitivity, specificity and accuracy. The formulas used to determine the measures are given below.

$$
\begin{gathered}
\text { Sens. }=\frac{T_{P}}{T_{P}+F_{P}} \\
\text { Spec. }=\frac{T_{N}}{T_{N}+F_{P}} \\
\text { Accu. }=\frac{T_{P}+F_{P}}{T_{P}+F_{P}+T_{N}+F_{N}}
\end{gathered}
$$

Where $T_{P}, T_{N}, F_{P}$ andF $F_{N}$ represents true positive, true negative, false positive and false negative respectively.
Fig. 5 displays the outcome of the presented approach representing the input image, its corresponding segmented and classified images.

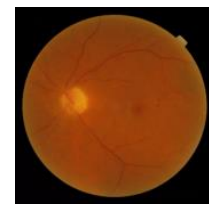

(a)

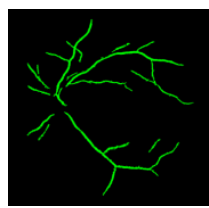

(b)

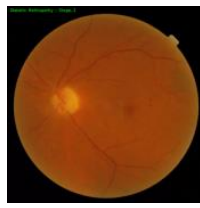

(c)
Fig. 5. a) Input Image b) Segmented Image c) Classified Image.

Table 2 also provides a detailed classification of diverse DR stages on the applied dataset. From this table, it is evident that the first column indicates the DR level, the second column represents the input image; third and fourth columns represent the segmented and classified images correspondingly. As shown in the table, the second row indicates that the input image is normal and the classified image also shows that the classified output is normal. It implies the effective classification of DR images by the presented model. Similarly, the third, fourth and fifth rows indicate the different stages of 2, 3 and 4 DR respectively. On all these applied DR images, the outcome exhibited that the presented approach effectively categorizes the DR into various stages.

An initial step to determine the classification performance is the generation of the confusion matrix. Table 3 shows the derived confusion matrix for the applied test images.

\subsection{Results and discussion}

Table 2. Classification of DR images under various stages

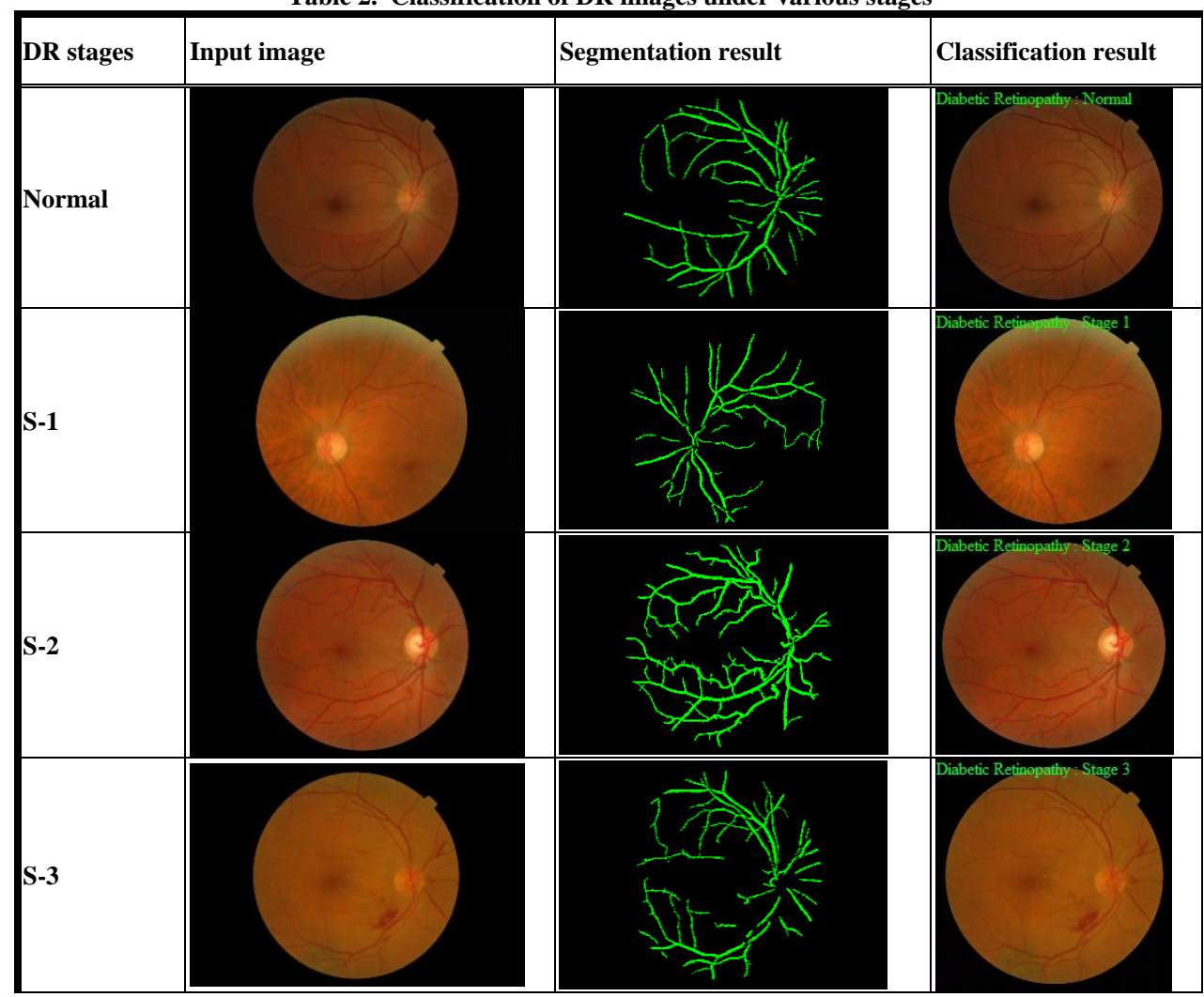


Table 3. Representation of Confusion Matrix for DR

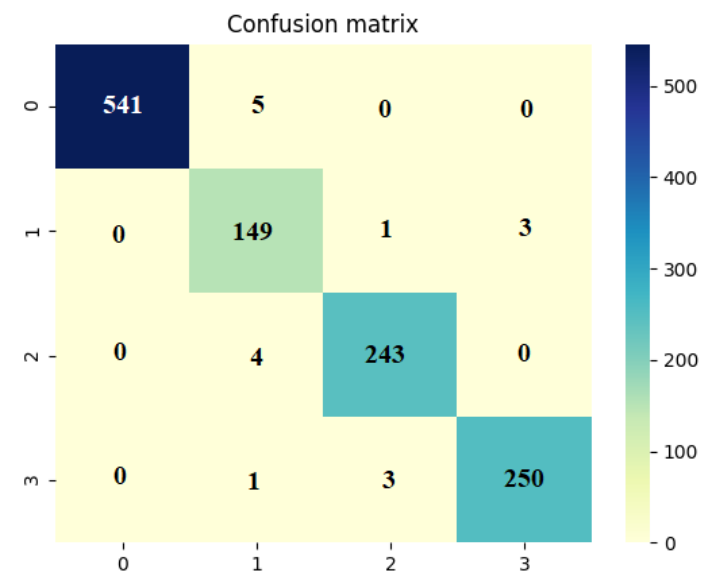

Table 3 is transformed to an understandable format in Table 4 representing the different stages of DR. The table values indicated that a total of 541 images are properly classified into normal stage and a total of 149, 243 and 250 images are effectively classified into three stages of 1,2 and 3 respectively.

Table 4. Confusion Matrix

\begin{tabular}{|l|l|l|l|l|l|}
\hline \multirow{2}{*}{ Labels } & \multicolumn{4}{|l|}{ Stages } & \multirow{2}{\text{Image}}{ Count } \\
\cline { 2 - 5 } & Healthy & S-1 & S-2 & S-3 & \\
\hline Healthy & 541 & 5 & 0 & 0 & 546 \\
\hline S-1 & 0 & 149 & 1 & 3 & 153 \\
\hline S-2 & 0 & 4 & 243 & 0 & 247 \\
\hline S-3 & 0 & 1 & 3 & 250 & 254 \\
\hline $\begin{array}{l}\text { Image } \\
\text { Count }\end{array}$ & 541 & 159 & 247 & 253 & 1200 \\
\hline
\end{tabular}

Then, the value of TP, TN, FP and FR for every stage of DR is computed and is placed in Table 5 and Fig. 6 . It will be helpful to determine the classification performance of the presented SDL model. The classifier results under different stages of DR are provided in Table 6 . The table values indicated that the normal images are classified with the maximum accuracy of 99.58, sensitivity of 100 and specificity of 100 . Similarly, the stage 1 images are also classified properly with the accuracy of 98.93 , sensitivity of 97.39 and specificity of 99.04 . Likewise, the stage 2 images are effectively classified with accuracy of 99.33, sensitivity of 98.38 and specificity of 99.58 . In the same way, the images under stage 4 are also effectively classified with the accuracy, sensitivity and specificity values of $99.41,98.42$ and 99.68 respectively.

Table 5. Manipulation from Confusion Matrix

\begin{tabular}{|l|l|l|l|l|}
\hline $\begin{array}{l}\text { DR } \\
\text { Level }\end{array}$ & Healthy & S-1 & S-2 & S-3 \\
\hline TP & 541 & 149 & 243 & 250 \\
\hline TN & 652 & 1034 & 940 & 933 \\
\hline FP & 0 & 4 & 4 & 4 \\
\hline FN & 5 & 10 & 4 & 3 \\
\hline
\end{tabular}

To further highlight the goodness of the presented model, a detailed comparison is made with the recently developed deep learning models and the results are provided in Table 7.
Table 6. Classifier results of presented model under various stages of DR

\begin{tabular}{|l|l|l|l|}
\hline $\begin{array}{l}\text { Input } \\
\text { Grades }\end{array}$ & Accu. & Sens. & Spec. \\
\hline Healthy & $\mathbf{9 9 . 5 8}$ & $\mathbf{1 0 0}$ & $\mathbf{9 9 . 2 4}$ \\
\hline S-1 & $\mathbf{9 8 . 8 3}$ & $\mathbf{9 7 . 3 9}$ & $\mathbf{9 9 . 0 4}$ \\
\hline S-2 & $\mathbf{9 9 . 3 3}$ & $\mathbf{9 8 . 3 8}$ & $\mathbf{9 9 . 5 8}$ \\
\hline S-3 & $\mathbf{9 9 . 4 1}$ & $\mathbf{9 8 . 4 2}$ & $\mathbf{9 9 . 6 8}$ \\
\hline
\end{tabular}

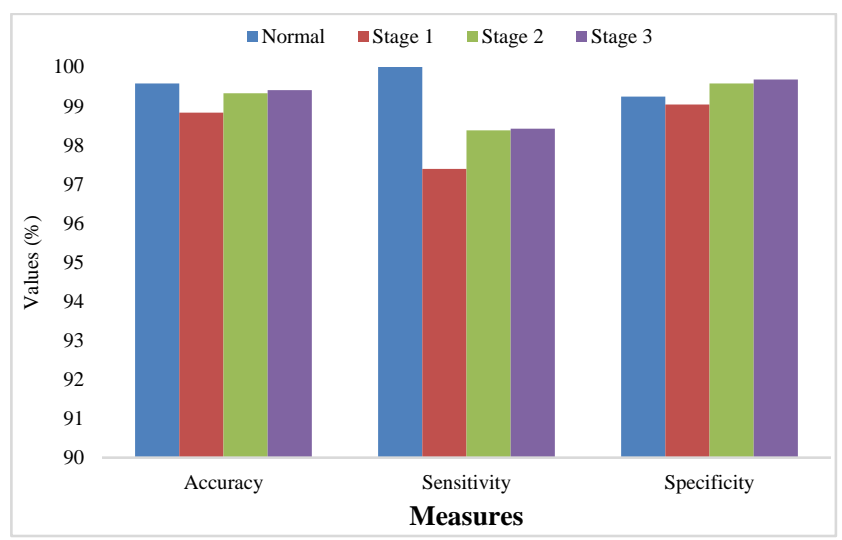

Fig. 6. Classifier results of presented model under various stages of DR.

Fig. 7also shows the comparison results of diverse methods on the applied DR image dataset. The table values indicated that the projected technique implies extraordinary classification performance. At the same time, the M-AlexNet model shows closer performance to the presented method, but fails to outperform it. In addition, the VggNet-s model shows better classification over the other methods in a significant way. However, it exhibits inferior performance over the presented and M-AlexNet models. In the same way, VggNet-16, VggNet-19 and GoogleNet offered almost identical classifier results. But, they are not as efficient as the presented SDL model. Furthermore, it is noted that the AlexNet model attains least classification with the minimum classifier accuracy of 89.75. From the table values, it is obvious that the presented SDL model shows excellent classification with the highest accuracy of 99.28 , sensitivity of 98.54 and specificity of 99.38 respectively.

Table 7. Comparison of classifier models under various measures

\begin{tabular}{|l|l|l|l|l|}
\hline Methods & Accuracy & Sensitivity & Specificity & CT (s) \\
\hline Proposed & 99.28 & 98 & 99 & 15.21 \\
\hline M-AlexNet & 96 & 92 & 97 & 16.53 \\
\hline AlexNet & 90 & 81 & 94 & 16.44 \\
\hline VggNet-s & 96 & 86 & 97 & 16.59 \\
\hline VggNet-16 & 93 & 91 & 94 & 17.14 \\
\hline VggNet-19 & 94 & 89 & 96 & 17.49 \\
\hline GoogleNet & 93 & 78 & 92 & 16.54 \\
\hline ResNet & 90 & 89 & 96 & 16.01 \\
\hline
\end{tabular}

To further ensure the goodness of the proposed model, a CT analysis is also made in Table 7. The table values indicated that the VggNet models acquire high CT over the other methods. Next to that, the AlexNet and M-AlexNet models offered moderate $\mathrm{CT}$ whereas the proposed method requires minimum CT of $15.21 \mathrm{~s}$ while classifying the fundus images. The overall experimentation proves the applicability of the presented model for the diagnosis of DR efficiently due to the following factors such as usage of histogram based segmentation and latest deep learning model. 


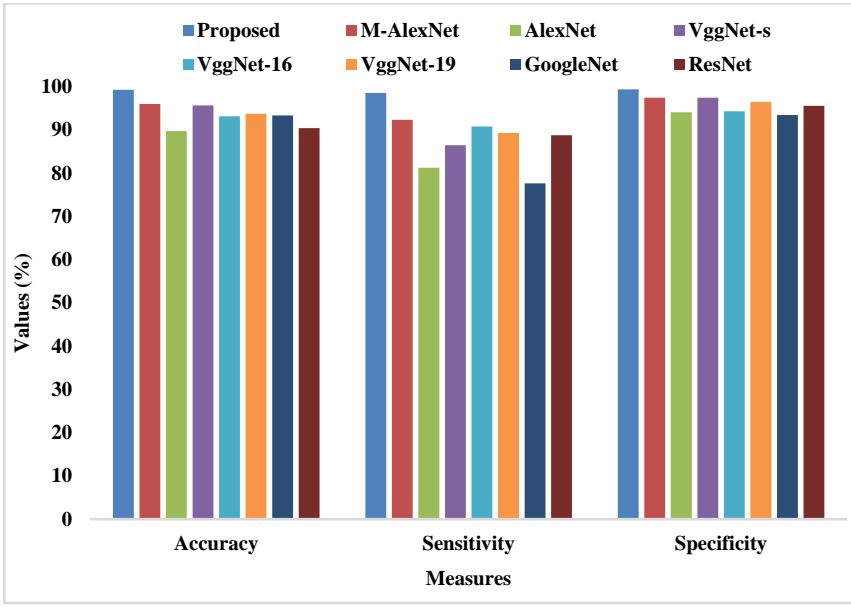

Fig. 7. Comparison of various classifier models.

\section{Conclusion}

In this paper, we have proposed a deep learning model referred to as SDL model for automated detection and classification of fundus DR images. This study intends to the classification of the DR fundus images with maximum detection rate. At the preprocessing stage, the unwanted noise present in the edges will be removed. Next, histogram-based segmentation takes place to extract useful regions from the image. Besides, SDL model is applied for the classification of DR fundus images into various stages. For the validation of the presented SDL model for the identification of DR, a benchmark MESSIDOR dataset is used. From the experimental values, it is observed that the projected method shows excellent classification with the highest accuracy of 99.28 , the sensitivity of 98.54 and specificity of 99.38 correspondingly. As a part of future scope, the presented model can be enhanced by the inclusion of filtering techniques to improve the quality of the images before processing. In addition, the proposed method can be extended by exploring more AlexNet and Inception techniques that could provide further improved performance by tuning the hyper parameters namely learning rate, epoch, dropout, number of hidden layers, momentum, activation function and so on.

\section{Acknowledgments}

This article has been written with the financial support of RUSAPhase 2.0 grant sanctioned vide Letter No. F. 24-51/2014-U, Policy (TNMulti-Gen), Dept. of Edn. Govt. of India, Dt. 09.10.2018.

\section{Conflict of Interest}

All the authors of the manuscript declared that there are no potential conflicts of interest.

\section{References}

1. Krizhevsky A, Sutskever I, Hinton GE. ImageNet classification with deep convolutional neural networks. Commun ACM 2017;60(May (6)):84-90.

2. Whiting DR, Guariguata L, Weil C, Shaw J. IDF diabetes atlas: global estimates of the prevalence of diabetes for 2011 and 2030. Diabetes Res ClinPract 2011;94(Dec (3)):311-21.

3. Hajeb Mohammad Alipour S, Rabbani H, Akhlaghi MR. Diabetic retinopathy grading by digital curvelet transform. Comput Math Methods Med 2012;2012:1-11.

4. SB S, Singh V. Automatic detection of diabetic retinopathy in non-dilated RGB retinal fundus images. Int J Comput Appl 2012;47(Jun (19)):26-32.
5. Singh N, Tripathi RC. Automated early detection of diabetic retinopathy using image analysis techniques. Int $\mathbf{J}$ Comput Appl 2010;8(Oct (2)):18-23

6. Cunha-Vaz BSPJG. Measurement and mapping of retinal leakage and retinal thickness - surrogate outcomes for the initial stages of diabetic retinopathy. Curr Med Chem-Immunol EndocrMetab Agents 2002;2(Jun (2)):91-108.

7. Anandakumar H, Umamaheswari K. Supervised machine learning techniques in cognitive radio networks during cooperative spectrum handovers. Cluster Comput 2017;20:1-11

8. Omar M, Khelifi F, Tahir MA. Detection and classification of retinal fundus images exudates using region based multiscale LBP texture approach. 2016 international conference on control, decision and information technologies (CoDIT), April; 2016.

9. Welikala RA, Fraz MM, Williamson TH, Barman SA. The automated detection of proliferative diabetic retinopathy using dual ensemble classification. Int J Diagn Imaging 2015;2(Jun (2))

10. Haldorai A, Ramu A, Chow C-O. Editorial: big data innovation for sustainable cognitive computing. Mob NetwAppl 2019;24(Jan):221-3.

11. Bhatkar AP, Kharat GU. Detection of diabetic retinopathy in retinal images using MLP classifier. 2015 IEEE international symposium on nanoelectronic and information systems, December; 2015.

12. Partovi M, Rasta SH, Javadzadeh A. Automatic detection of retinal exudates in fundus images of diabetic retinopathy patients. J Anal Res Clin Med 2016;4(May (2)):104-9.

13. Xu, Y., Mo, T., Feng, Q., Zhong, P., Lai, M., Eric, I., Chang, C., 2014. Deep learning of feature representation with multiple instance learning for medical image analysis, in: Proceedings of IEEE International Conference on Acoustics, Speech and Signal Processing (ICASSP), pp. 1626-1630

14. Shen, W., Zhou, M., Yang, F., Yu, D., Dong, D., Yang, C., Zang, Y., Tian, J., 2017. Multi-crop convolutional neural networks for lung nodule malignancy suspiciousness classification. Pattern Recognition 61, 663-673.

15. Zhou, T., Thung, K.H., Zhu, X. and Shen, D., 2019. Effective feature learning and fusion of multimodality data using stage-wise deep neural network for dementia diagnosis. Human brain mapping, 40(3), pp.1001-1016.

16. Fan, J., Cao, X., Yap, P.T. and Shen, D., 2019. BIRNet: Brain image registration using dual-supervised fully convolutional networks. Medical image analysis, 54, pp.193-206.

17. Zhou, G., Zhao, Q., Zhang, Y., Adal1, T., Xie, S. and Cichocki, A., 2016. Linked component analysis from matrices to high-order tensors: Applications to biomedical data. Proceedings of the IEEE, 104(2), pp.310-331.

18. Zhang, Y., Zhang, H., Chen, X., Liu, M., Zhu, X., Lee, S.W. and Shen, D., 2019. Strength and similarity guided group-level brain functional network construction for MCI diagnosis. Pattern Recognition, 88, pp.421-430

19. Mohamed Elhoseny, K. Shankar, J. Uthayakumar, "Intelligent Diagnostic Prediction and Classification System for Chronic Kidney Disease", Nature Scientific Reports, July 2019. In Press. https://doi.org/10.1038/s41598-019-46074-2

20. K. Shankar, Lakshmanaprabu S.K, Ashish Khanna, Sudeep Tanwar, Joel J.P.C.Rodrigues, Nihar Ranjan Roy, "Alzheimer detection using Group Grey Wolf Optimization based features with convolutional classifier", Computers \& Electrical Engineering, Volume 77, Pages 230-243, July 2019.

21. Mohamed Elhoseny, K.Shankar, "Optimal Bilateral Filter and Convolutional Neural Network based Denoising Method of Medical Image Measurements", Measurement, Volume 143, Pages 125-135, September 2019.

22. Lakshmanaprabu S.K, Sachi Nandan Mohanty, K. Shankar, Arunkumar N, Gustavo Ramireze, "Optimal deep learning model for classification of lung cancer on CT images", Future Generation Computer Systems, Volume 92, Pages 374-382, March 2019

23. K. Shankar, Mohamed Elhoseny, Lakshmanaprabu S K, Ilayaraja M, Vidhyavathi RM, Majid Alkhambashi, "Optimal feature level fusion based ANFIS classifier for brainMRI image classification", Concurrency and Computation: Practice and Experience, August 2018.

24. http://www.adcis.net/en/third-party/messidor/ 